\title{
Technologically Mediated Intimate Communication: An Overview and Future Directions
}

\author{
Elham Saadatian ${ }^{1}$, Hooman Samani ${ }^{2}$, Arash Toudeshki $^{3}$, and Ryohei Nakatsu ${ }^{4}$ \\ 1 Interactive and Digital Media Institute, National University of Singapore \\ 2 Department of Electrical Engineering, National Taipei University, Taiwan \\ 3 Department of Electrical and Electronic Engineering, University Putra Malaysia \\ 4 Department of Electrical and Computer Engineering, \\ National University of Singapore \\ Keio-NUS CUTE Center, NUS \\ elham@nus.edu.sg
}

\begin{abstract}
Emerging field of intimate computing relates to the technologies that aim to mediate affective communication across distance. Conventional telecommunication media are originally designed for collaborations and task oriented goals with the poor support of intimate experiences. Contemporary lifestyle changes leaded to design and adoption of technologies in support of long distance relationships. The present work is a study of existing prototypical systems and related conceptual studies in this realm of study. Their design perspective, mechanism and human factors are described. Challenges coupled to this domain are studied and future research directions are proposed.
\end{abstract}

Keywords: Telepresence, Mediated intimacy, Telematic emotional communication, User interface, Perspective.

\section{Introduction}

Intimacy is defined by social scientists as cognitive and emotional expressiveness, support, engagement, and physical contact [1. Maintaining intimacy in the relationship is emphasized as a necessary factor of the romantic relationship [2]. However, increase of Long Distance Relationships (LDR), and poor functionality of current telecommunication system hampers the convergence of intimacy across distance.

Conventional technologies for mediated remote communication are originally designed for collaboration, teleoperation, and task oriented activities which are optimized for efficient exchange of information. This trend compromises the noninformative dimension of interaction. Therefore, intimate computing increasingly gained the attention of Human Computer Interaction (HCI) and Human Robot Interaction (HRI) researchers to support LDR and has opened a new area of study known as intimate computing. It resulted in a considerable amount of 
studies and prototypes, by adopting the metaphor of remote or mediated intimacy. In these technologies the goal is promoting social bond rather than any information or data transition. The pioneering works in this area are three prototypical systems namely " Feather, Scent, and Shaker developed by Strong \& Gaver [3] which affords remote intimate communication by providing ambient representation of the distant partner. It gave a new insight in telecommunication technologies. This paper takes and opportunity to survey the current state-ofthe-art in intimate computing. It describes research and prototypes developed to date, and provide suggestions for future directions of research in this area.

\section{Human Factor Elicitation for Design of Intimate Medium}

Intimate communications are distinct form typical communications studied by HCI researchers, such as relationships amongst friends or colleagues [4]. Challenges on studying intimacy arises from their ephemeral nature, low informational content and emotional significant, self-disclosure and privacy, unsaid interactions and idiosyncratic nature. Also there is no predefined language for its description [5]. Since intimate behaviors are strikingly nuanced and often subtly vague to outsiders, involvement of users is unavoidable in design of its supporting technologies. One area of research in intimate computing relates to human factor elicitation and user experience of these technologies.

One of the approaches is using technology probes. For example: Lottridge et al. 6] explored the design space for remote intimate communication between couples. They realized the potential to draw on the daily routine "empty moments" of couples. Ambiguity, aesthetic, continuity, asymmetry, and movability were comforted by users as design space for sharing empty moments.

OB́rien \& Mueller [7] applied technology probe to investigate when partners in close relationships would want tactile exchange through holding hands while apart. However the experiment was not successful since the probe was too simple to encourage couples to use it. They realized a probe should be simple but aesthetically well designed to encourage participant to use it. Kaye \& Goulding [ developed personalized probes for long term study through the users proposed sketches. They studied how objects were used and how changed their communication pattern. This study was based on the advantages of personal over mass communication in the context of intimacy.

Another perspective focuses on scenario based design. For example, Battarbee et al. 9] tried to design in support of intimacy within an urban environment. It was done through observations within the city and developing scenarios. King [10] explored how people use interactive technologies in their relationships through legitimating the participates to explore the possible future, instead of being restricted to current technology.

Other studies on design space are: King \& Forlizzi [11] used interview, web based diary study and photo journal. They found possibilities for emotional connectedness by designing reflective and slow interfaces which are linked to the 
sense of place. Pace et al. 12 studied virtual world as a source of intimacy and found permeability across virtual and real worlds, mundane as origin of intimacy, reciprocity and exchange, and temporality in shaping intimate experience. Gooch \& Watts 13 proposed a framework to formalize the design space for intimate communication devices. They have highlighted the 6 factor of Personalization, Sensory Medium, Effort, Openness of the System, Metaphor of Use, and Fleeting vs Realized Output. These factors are based on the study of previous developed devices and more study is needed for validation and refining the framework.

There has been several attempts in exploring the effect of physiological and nonverbal signals on perception of intimate connections. For instance: Gooch \& Watts 14 proved the impact of heat as an aspect of touch using thermal hug belt. Carpe Diem [15] proved the effect of eye contact in intimacy. Slovák et al. [16] Investigated people perception on heart rate feedback. They found heartrate feedback an affective connectedness signals as well as connector. In another study Janssen et al. [17] empirically proved the possibility of using heartbeat transition as an intimate signal through self-report and behavioral monitoring in an immersive virtual environment.

These are not the only attempts in studying intimate computing through user, however these are studies which dominantly focused on user perception. Other researches have also involved users in the system design and evaluation into some extents.

\section{Prototypes for Mediated Intimacy}

The design space for remote intimate couples has largely been populated with technologies that support and mediate intimacy via abstracted presence [18 19]. Gaver categorizes these technologies into two groups, those which mediate intimate behaviors and those which provoke intimate reactions [3]. The first group attempts to mediate intimate feelings or actions through reproduction, manifestation or imitation of them, using technology. Whilst the second group concentrates on evoking reactions instead of explicit expressions [20. Gaver [5] has identified three common features in most of these technologies: use of evocative materials; use of poetic mappings rather than didactic metaphors; and reliance on physical materials.

Fig. 1 shows the relative distribution of some of these technologies that will be described in the following sections. They are classified based on degree of holism and realness versus abstract and poetic presence that they convey.

\subsection{Technologies That Mediate Intimate Behaviors}

In this group commonly pair of coupled interfaces transmit the intimate behavior requiring near body presence. Whereas the interface acts on behalf of the remote person to literally or symbolically reproduced the intimate expressions. In literal approach the intimate action is directly reproduced. Whilst in symbolic (poetic) simulation, intimate behavior is mapped to another form. Attempts on 


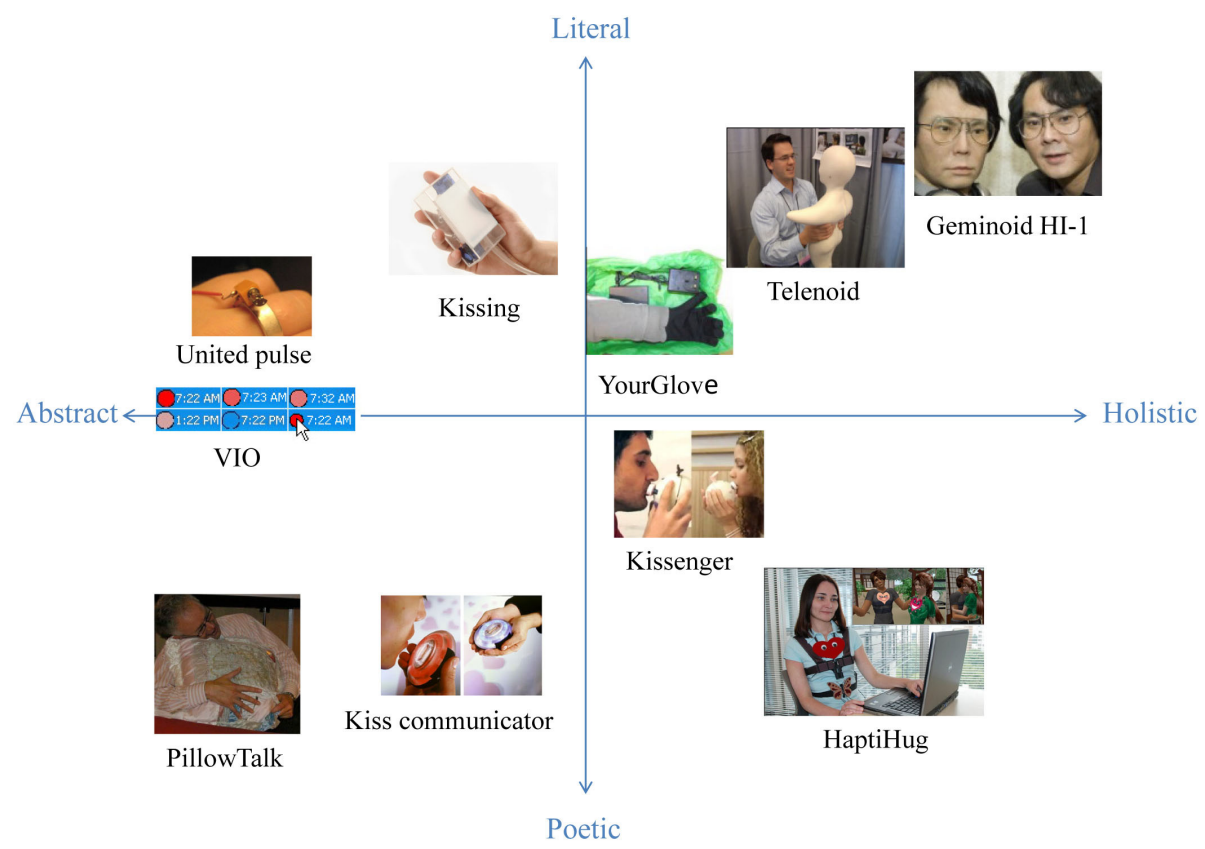

Fig. 1. Relative distribution of some of the telepresence technologies

this category includes different types of remote haptic communications such as hugging, kissing, grasping, shaking hand, hand holding. Other physical interactions such as, whispers, sound of heartbeat and body heat of significant others sleeping beside are in this category.

\subsection{Technologies That Provoke Intimate Reactions}

One perspective on intimate computing is implicit affective communication. In this approach abstract interfaces can convey the sense of presence in absence in an ambient way. They do not transmit any intimate behavior however they support emotional aspects of intimacy and provoke intimate reaction without explicit expression.

Ephemeral and Poetic Interactions. There has been several works that provoke intimacy through ephemeral interactions. The earliest of this type are "Feather, Scent, and Shaker" envisioned by Strong and Gaver [3. They are paired devices that implicitly aware users when their remote partner thinks of them. In "Feather" interaction with a picture frame causes the feather drift in the air. Shaker facilitates the exchange of tactile gestures through a simple remote force feedback mechanism. And scents actuates ephemeral aroma at home when the traveling partner interacts with a photo frame. These prototypes convey romantic sensation through serendipitous and ephemeral attributes of interfaces. 


\begin{tabular}{|c|c|c|c|}
\hline Message & prototype & medium design & Stimulation \\
\hline Kiss & $\begin{array}{l}\text { Intimate mobiles [22] } \\
\text { kiss communicator [23] } \\
\text { Kissenger [24] } \\
\text { CheekTouch [25], [26] } \\
\text { Hkiss [27] } \\
\end{array}$ & $\begin{array}{l}\text { direct telepresence on mobile } \\
\text { Symbolic mapping } \\
\text { direct mapping on telepresence robot } \\
\text { differnt vibration patterns } \\
\text { haptic feedback from 3D avatar } \\
\end{array}$ & $\begin{array}{c}\text { hand loop, heat, wet sponge, airjet } \\
\text { squeeze and blow } \\
\text { vibration } \\
\text { phone vibration and audio } \\
\text { vibration on belt }\end{array}$ \\
\hline Hug & $\begin{array}{c}\text { The Hug [28] } \\
\text { Hug over a distance [29] } \\
\text { Huggy pajama [30] } \\
\text { HaptiHug [31] } \\
\text { Second Life HugMe [32] }\end{array}$ & $\begin{array}{c}\text { via telepresence robot } \\
\text { through wearable vest } \\
\text { wearable hug jacket and hug trigger device } \\
\text { autodetect hug keyword,visualization and haptics } \\
\text { multimodal hug input }\end{array}$ & $\begin{array}{l}\text { lights, vibration patterns, heat,sounds. } \\
\text { air-inflation } \\
\text { air pressure } \\
\text { robotic hand pressure } \\
\text { visuotactile on jacket and avatar }\end{array}$ \\
\hline Co-Sleeping & $\begin{array}{l}\text { Sensing Beds [33] } \\
\text { Aura [34] }\end{array}$ & $\begin{array}{l}\text { position tracking via mattress sensors } \\
\text { sleep pattern sesning }\end{array}$ & $\begin{array}{l}\text { slow heating } \\
\text { music corresponding to pattern }\end{array}$ \\
\hline Hand Touching & $\begin{array}{c}\text { Tele-handshake [35] } \\
\text { YourGlove [36] } \\
\text { HotHands and HotMits [37] } \\
\text { Feelybean [38] } \\
\text { Stroking device } \\
\text { while holding hands [39] } \\
\text { The Withe Stone [40] } \\
\text { VibroBod [41] }\end{array}$ & $\begin{array}{l}\text { shared virtual interface and hapticcs } \\
\text { paired robotic hands } \\
\text { personalized hand shape } \\
\text { augment touch to skype } \\
\text { exchange stroke gesture } \\
\text { paired stone } \\
\text { interface detects gesture/voice }\end{array}$ & $\begin{array}{c}\text { feedback through phantom } \\
\text { hold and grip } \\
\text { thermal insulation } \\
\text { vibration } \\
\text { servo motors under sponge } \\
\\
\text { beep sound and heat } \\
\text { warmth and pose }\end{array}$ \\
\hline Biological Signals & $\begin{array}{l}\text { Mobile Feelings [42] } \\
\text { united-pulse [43] }\end{array}$ & $\begin{array}{l}\text { heart beat/breath sharing } \\
\text { heartbeat share } \\
\text { user heartbeat measured }\end{array}$ & $\begin{array}{l}\text { LED blinking rhythmic pulses/ micro-ventilator } \\
\text { ring vibration }\end{array}$ \\
\hline Touch Gestures & $\begin{array}{c}\text { Tug } \mathrm{n}^{*} \text { Talk [44] } \\
\text { Poke [45] } \\
\text { The ComSlipper [46] } \\
\text { FootIO [47] } \\
\text { InTouch [48] } \\
\text { PillowTalk [49] }\end{array}$ & $\begin{array}{l}\text { belt-buckle with chains } \\
\text { surface augmented on mobile } \\
\text { foot posture sensed emotion } \\
\text { foot-stool } \\
\text { paird rollers } \\
\text { pillow detects hand gestures }\end{array}$ & $\begin{array}{c}\text { chain moves } \\
\text { inflation } \\
\text { vibration/warmth/light } \\
\text { different vibrations } \\
\text { vibration } \\
\text { ambient method }\end{array}$ \\
\hline Holistic Presence & $\begin{array}{c}\text { Geminoid HI-1 [50] } \\
\text { Elfoid phone [51] } \\
\text { Mebot [52] } \\
\text { Callo and Cally [53] }\end{array}$ & $\begin{array}{l}\text { Realistic humanoid robot } \\
\text { miniature, generic humanoid robot } \\
\text { robot and cellphoen display } \\
\text { robot and cellphoen display }\end{array}$ & $\begin{array}{l}\text { exact voice and some moves } \\
\text { exact voice and some moves } \\
\text { facial expression and gestures } \\
\text { walk, dance, facila expressions }\end{array}$ \\
\hline
\end{tabular}

Fig. 2. Relative distribution of some of the telepresence technologies

"Hintouch" 21] is a Two-Way Ambient Communication prototype with support of context information around picture.

"LumiTouch" 22] is a pair of picture frames with the photo of remote users on each other's desks which are used as interface. When the sender squeezes the picture frame, her partner's picture frame displays area illuminates corresponding with input squeeze intensity. Different combination of of light intensities, colors, and pulses are decided by couples as a private language for their interaction.

Also "SmallConnection" 23. expresses faint information such as light, wind and touch using robotic technology. Light symbolizes presence by synchronically turning on and off between two distant homes. Winds detects the sound in remote place and spins in sync with it. And touch is transmitted by synchronized movement of paired buttons. "VIO" Virtual Intimate Objects (VIO) [24] transmits one bit message by clicking on a virtual circle on a task bar that brightens the remote persons' circle.In another study on VIO 25] users were evaluated using a logbook including open ended questions regarding the context of use. This Study also revealed that this simple interaction has a rich interpretation which suggest the importance of usage context in remote communication technologies.

Co-presence through Hybrid Sensory Interaction. There has been several prototypes that express intimacy through hybrid sensory mode. Such as: "Keep in touch" 26] that facilitates playful, visuotactile interaction by 'fabric screen'. Each persons' screen displays an out-of-focus video of his/her counterpart, that comes to focus after touching the fabric. "ComTouch" 27] augments touch to voice communication by sending vibrotactile feedback on the mobile phone during conversation. "Shake2Talk" 28] is a mobile messaging system that facilitates sending audio-tactile messages by SMS. Messages are composed by four gestures 
of stroke, tap, flick which are mapped to different audio-tactile message and played to the sender as they make the gesture. User evaluation showed it was most used by couples living apart.

Shared Experience. Bhandari\& Bardzell [29] proposed four design concepts around these them: "Matchus board" and "Together aquarium" were based on the theme of experience sharing. In which the first one facilitates experience sharing by synchronized drawing on a board using multi-user touch input and output devices. And the later through coordination on taking care of online virtual aquarium. The second pair were based on the theme of awareness namely "Cancan" and "Audible gifts". Cancan was a button augmented on a watch which corresponds to "cannot be available now". Audible gift was inspired by significance of "letters" since they have tangibility, thoughtfulness, and require effort which is a pair of microphone and speaker for sending five minutes message.

"Lovers' Cup" 30] explores the idea of conveying the feelings of co-drinking as a communication channel for a couple in physically different places. Amount of liquid in each cup is shown in the partners' cup with LEDs and shaking the cups vibrate its remote pair.

Simple Awareness. "BuddyClock" 31] is a network-enabled alarm clock, which can share alarm status with alarm clocks within a social group. It is predicted such natural status sharing may enhance social awareness, facilitate self-reflection and intimacy. In case of distant romantic couples after a few days their sleeping pattern was synchronized.

"CoupleVIBE" 32 is an awareness unobtrusive privacy-friendly, communication channel on mobile. It shares location information of remote partners by sending specialized touch cues when users moves to each location. This medium allows them to keep updated without being distracted from their daily activity.

"Personal portraits" rises or dims a remote photo frame based on the existence or absence of key ring, which is ambience representation of presence at home. "Light sculptures" uses light bulbs to show presence at home. And in "SoftAir Communication" pressure and movements are detected through embedded sensors in the chair when they are touched and show the presence of remote person by embedded lights and sounds on the touched surface [33].

Effort and Mutual Exchange. Another abstract approach which affords selfdisclosure, communicates moods and shows effort and individualization is "sharing emotion through self composed melodies" [34]. This system allows users to compose and share melodies via mobile phones. It synchronically shares emotional state of the sender. They founded self composed melodies have strong impact on receiver which is similar to the effect of crafted piece of art offered to a beloved person.

"Lovers' box" [35] is a digital artifact that aims to engage couples in reflection on their relationship. The role of refection which is through creation, exchange and sharing is examined using digital artifacts. Feeling communication interface was a box that could exchange self-created video messages between couples. The 
other factors that supported intimate interaction were being more customizable than text, private sharing, and giving \& receiving. It also has ambiguity since it allows the users to interpret and analyze the message.

\section{Fictitious Co-habiting through Sharing Everyday Artifacts and Ob-}

jects. Another group of prototypes use the existing and adapting existing artifacts as a communication medium instead of creating a completely new device. This theme is initiated by Dodge through a prototype named "The bed" 36]. By equipping the pillows with heating pads and vibrating motors presence and heartbeat of the remote person is symbolized.

"Peek-a-drawer" [37] facilitates virtually sharing drawers among remote family members. In this device when a user put something in the drawer its photo is taken and appears in the remote drawer. "Habitat" 38 is a series of joined furniture for background awareness between distant couples. The initial prototype consists of two, networked coffee table whereas each station consists of a computer, RFID tag reader and a video projector. Objects placed on the table are sensed by the RFID reader and its corresponding representation is projected on the remote table. When objects are removed their representation fades gradually.

"SyncDecor" 39] informs couples of each other's activity by synchronizing daily appliances. This system can remotely synchronize lamps lightness, trash door open, TV channel and smell between remote couples. "Magic Sock Drawer" 40] enables to create digital hand-drawn or typed messages and then automatically produce the physical printed version in the remote users' drawer. The system design supports four intimate computing design concepts including: exchange between dyads, personalization, tangibility and location sharing.

"Digital Selves" proposed by Grivas 41] proposes fictitious merge of the homes of remote couples. This system recognizes the positions of electronic objects inside the two disparate homes of a couple living apart. The advantage over simple ambient communication is adding spatiality and creating sense of place.

\section{Discussions and Recommendations for Future Research}

In this paper we have attempted to present a broad outline of the field of intimate computing, including relevant conceptual descriptions, human factor elicitation, technical mechanism, and current design trends, fueled by psychological attributes, as well as trends in affective computing, social technologies, and telepresence. The prototypical systems and design perspectives reviewed in the present paper have set the stage for future studies on intimate computing by inspiring researchers, designers, and developers by providing description of the state of the art.

However, it seems that in design investigation and point solutions, there is still ambiguity in approach that best support LDR. Deeper conceptual understanding on the nature of intimacy and practical experiments on the effects of mediated intimacy is needed. Due to the private and delicate nature of intimacy conventional user need exploration techniques such as ethnography are 
not efficient enough. There have been several attempts in use of cultural probes, but since the current attempts are still rudimentary, more encouraging and well designed cultural probes are still needed.

Although the field of intimate computing is largely populated by technologies that facilitate experience sharing, they have likeness and monotony and very naive approach to love and relationships 42. Therefore technologies and design ideas that support different subjective love languages are needed.

To date very few objective evaluation techniques of the systems are available beyond the simple descriptions from users or quantitative analysis with few participants. Studying the long term effect of intimae interaction through developed mediums and benchmarking among different suggested designs is rarely experienced. We recommend more detail studies on the user acceptance of such novel trend of communication, and also studying potential cultural, gender and age differences in the degrees of acceptance and ethical issues. What the relatively new area of intimate computing would benefit most from at this early stage is more studies that put the assumed design decisions, described in the reviewed literature to the test and practice in the real world application.

Study on unmediated intimate communication, reveals unmet research questions. For instance an interesting question is whether people hold the same expectancies about the use of mediated intimacy as about the unmediated one. Whether or not the mediated has the same physiological and psychological effects. For instance in real-life scenario people have different reactions to opposite sex and to their own partner compared to same sex and stranger. Exploring the design solutions and engineering ideas that recreate the same sensation in mediated environments is also recommended.

Current technologies still do not support the same natural sensation of real intimate communication. Studies on real interaction offers a good solution to explore the necessary technical and design improvements. Experimental studies on physical properties of real intimate interactions could help to figure out the physical properties (e.g., required actuation amount, pattern) which may lead to more organic sensation.

Exploration on alternative solutions with the same physiological or perceived effect instead of literal simulation of intimate behaviors is suggested. For instance, approaches such as rubber hand illusion 43 . could be adapted to provide the perception that the medium acting on behalf is the extension of self.

Also physical close interactions, causes the release of chemicals such as oxytocin and serotonin that help to feel happy and connected 44. Studies on alternate technologies that stimulate brain for the same reaction could contribute to this field. Also since the nature of this study is multidisciplinary involvement of people from different fields can be promising.

Common theme of intimate computing technologies is pairing abstract devices that connected through internet and enable simple interactions. However, a holistic telepresence medium that provokes or expresses intimacy is not yet developed. 
In intimate communication systems, there is a move towards investigating the use of touch. In current systems, it is usually done through simple vibration, pressures or other forms of actuation. Development of high fidelity mediums through advanced control of tactile stimulation algorithms, non-invasive sensing technologies or machine leaning is still needed.

Current telecommunication mediums such as video conferencing compromise the non-verbal signal of eye-gaze direction. Although there has been very few attempts in this area 45. they are still in their infantry. Technical improvements and integration in intimate computing interfaces could be promising. Another suggested research direction is development of intelligent agents to interfere and advice in the relationships instead of simply transmitting the nonverbal cues. Also improvement in usability (exp., non-disruptiveness, embedding in current communication medium instead of separate device, and portability.) is suggested.

\section{Conclusion}

To sum up, the studies reviewed in the present paper show variety of promising design perspectives of mediated intimacy, ranging from once in a while communicating a bit of data 24, to a complex telepresence robot [46]. However, to date, the developed interfaces are still prototype and needs cutting edge technical, design and usability improvements to be empirically applied in real-life scenarios. Doing so has consequence for the progress of the field, for science, commerce, and social health.

Acknowledgement. This research is supported by the Singapore National Research Foundation under its International Research Center Keio-NUS CUTE Center @ Singapore Funding Initiative and administered by the IDM Program Office.

\section{References}

1. Moss, B., Schwebel, A.: Defining intimacy in romantic relationships. Family Relations, 31-37 (1993)

2. Cheal, D.: Showing them you love them: gift giving and the dialectic of intimacy. The Sociological Review 35(1), 150-169 (2011)

3. Gaver, B.: Provocative awareness. In: Computer Supported Cooperative Work (CSCW), vol. 11(3), pp. 475-493 (2002)

4. Kjeldskov, J., Gibbs, M., Vetere, F., Howard, S., Pedell, S., Mecoles, K., Bunyan, M.: Using cultural probes to explore mediated intimacy. Australasian Journal of Information Systems 11(2), 102-115 (2007)

5. Vetere, F., Gibbs, M., Kjeldskov, J., Howard, S., Mueller, F., Pedell, S., Mecoles, K., Bunyan, M.: Mediating intimacy: designing technologies to support strong-tie relationships. In: Proceedings of the SIGCHI Conference on Human Factors in Computing Systems, pp. 471-480. ACM (2005) 
6. Lottridge, D., Masson, N., Mackay, W.: Sharing empty moments: design for remote couples. In: Proceedings of the 27th International Conference on Human Factors in Computing Systems, pp. 2329-2338. ACM (2009)

7. O'Brien, S., Mueller, F.: Holding hands over a distance: technology probes in an intimate, mobile context. In: Proceedings of the 18th Australia Conference on Computer-Human Interaction: Design: Activities, Artefacts and Environments, pp. 293-296. ACM (2006)

8. Kaye, J., Goulding, L.: Intimate objects. In: Proceedings of the 5th Conference on Designing Interactive Systems: Processes, Practices, Methods, and Techniques, pp. 341-344. ACM (2004)

9. Battarbee, K., Baerten, N., Hinfelaar, M., Irvine, P., Loeber, S., Munro, A., Pederson, T.: Pools and satellites: intimacy in the city. In: Proceedings of the 4th Conference on Designing Interactive Systems: Processes, Practices, Methods, and Techniques, pp. 237-245. ACM (2002)

10. Howard, S., Vetere, F., Gibbs, M., Kjeldskov, J., Pedell, S., Mecoles, K., Bunyan, M., Murphy, J.: Mediating intimacy: digital kisses and cut and paste hugs. In: Proceedings of BCSHCI 2004, Leeds, UK (2004)

11. King, S., Forlizzi, J.: Slow messaging: intimate communication for couples living at a distance. In: Proceedings of the 2007 Conference on Designing Pleasurable Products and Interfaces, pp. 451-454. ACM (2007)

12. Pace, T., Bardzell, S., Bardzell, J.: The rogue in the lovely black dress: Intimacy in world of warcraft. In: Proceedings of the 28th International Conference on Human Factors in Computing Systems, pp. 233-242. ACM (2010)

13. Gooch, D., Watts, L.: A design framework for mediated personal relationship devices. In: Proceedings of the 25th BCS Conference on Human-Computer Interaction, pp. 237-242. British Computer Society (2011)

14. Gooch, D., Watts, L.: Communicating social presence through thermal hugs. In: SISSI 2010, p. 11 (2010)

15. Väänänen-Vainio-Mattila, K., Suhonen, K., Gonsalves, T., Schrader, M., Järvenpää, T.: Carpe diem: exploring user experience and intimacy in eye-based video conferencing. In: Proceedings of the 10th International Conference on Mobile and Ubiquitous Multimedia, pp. 113-122. ACM (2011)

16. Slovák, P., Janssen, J., Fitzpatrick, G.: Understanding heart rate sharing: towards unpacking physiosocial space. In: Proceedings of the 2012 ACM Annual Conference on Human Factors in Computing Systems, pp. 859-868. ACM (2012)

17. Janssen, J., Bailenson, J., IJsselsteijn, W., Westerink, J.: Intimate heartbeats: Opportunities for affective communication technology. IEEE Transactions on Affective Computing (99), 1 (2010)

18. Branham, S., Harrison, S., Hirsch, T.: Expanding the design space for intimacy: supporting mutual reflection for local partners. In: Proceedings of the Designing Interactive Systems Conference, pp. 220-223. ACM (2012)

19. Branham, S., Harrison, S.: Designing for collocated couples. Connecting Families, 15-36 (2012)

20. Davis, H., Skov, M., Stougaard, M., Vetere, F.: Virtual box: supporting mediated family intimacy through virtual and physical play. In: Proceedings of the 19th Australasian Conference on Computer-Human Interaction: Entertaining User Interfaces, pp. 151-159. ACM (2007)

21. Petersen, M.G., Hansen, A.B., Nielsen, K.R., Gude, R.: HOMEinTOUCH designing two-way ambient communication. In: Aarts, E., Crowley, J.L., de Ruyter, B., Gerhäuser, H., Pflaum, A., Schmidt, J., Wichert, R. (eds.) AmI 2008. LNCS, vol. 5355, pp. 44-57. Springer, Heidelberg (2008) 
22. Chang, A., Resner, B., Koerner, B., Wang, X., Ishii, H.: Lumitouch: an emotional communication device. In: CHI (2001)

23. Ogawa, H., Ando, N., Onodera, S.: Smallconnection: designing of tangible communication media over networks. In: Proceedings of the 13th Annual ACM International Conference on Multimedia, pp. 1073-1074. ACM (2005)

24. Kaye, J., Levitt, M., Nevins, J., Golden, J., Schmidt, V.: Communicating intimacy one bit at a time. In: CHI 2005 Extended Abstracts on Human Factors in Computing Systems, pp. 1529-1532. ACM (2005)

25. Kaye, J.: I just clicked to say i love you: rich evaluations of minimal communication. In: CHI 2006 Extended Abstracts on Human Factors in Computing Systems, pp. 363-368. ACM (2006)

26. Motamedi, N.: Keep in touch: a tactile-vision intimate interface. In: Proceedings of the 1st International Conference on Tangible and Embedded Interaction, pp. 21-22. ACM (2007)

27. Chang, A., O'Modhrain, S., Jacob, R., Gunther, E., Ishii, H.: Comtouch: design of a vibrotactile communication device. In: Proceedings of the 4th Conference on Designing Interactive Systems: Processes, Practices, Methods, and Techniques, pp. 312-320. ACM (2002)

28. Brown, L., Sellen, A., Krishna, R., Harper, R.: Exploring the potential of audiotactile messaging for remote interpersonal communication. In: Proceedings of the 27th International Conference on Human Factors in Computing Systems, pp. 1527-1530. ACM (2009)

29. Bhandari, S., Bardzell, S.: Bridging gaps: affective communication in long distance relationships. In: CHI 2008 Extended Abstracts on Human Factors in Computing Systems, pp. 2763-2768. ACM (2008)

30. Chung, H., Lee, C., Selker, T.: Lover's cups: drinking interfaces as new communication channels. In: CHI 2006 Extended Abstracts on Human Factors in Computing Systems, pp. 375-380. ACM (2006)

31. Kim, S., Kientz, J., Patel, S., Abowd, G.: Are you sleeping?: sharing portrayed sleeping status within a social network. In: Proceedings of the 2008 ACM Conference on Computer Supported Cooperative Work, pp. 619-628. ACM (2008)

32. Bales, E., Li, K., Griwsold, W.: Couplevibe: Mobile implicit communication to improve awareness for (long-distance) couples. In: Proceedings of the ACM 2011 Conference on Computer Supported Cooperative Work, pp. 65-74. ACM (2011)

33. Tollmar, K., Junestrand, S., Torgny, O.: Virtually living together. In: Proceedings of the 3rd Conference on Designing Interactive Systems: Processes, Practices, Methods, and Techniques, pp. 83-91. ACM (2000)

34. Shirazi, A., Alt, F., Schmidt, A., Sarjanoja, A., Hynninen, L., Häkkilä, J., Holleis, P.: Emotion sharing via self-composed melodies on mobile phones. In: Proceedings of the 11th International Conference on Human-Computer Interaction with Mobile Devices and Services, p. 30. ACM (2009)

35. Thieme, A., Wallace, J., Thomas, J., Le Chen, K., Kramer, N., Olivier, P.: Lovers' box: Designing for reflection within romantic relationships. International Journal of Human-Computer Studies (2010)

36. Dodge, C.: The bed: a medium for intimate communication. In: CHI, vol. 97, pp. 22-27 (1997)

37. Siio, I., Rowan, J., Mynatt, E.: Peek-a-drawer: communication by furniture. In: CHI 2002 Extended Abstracts on Human Factors in Computing Systems, pp. 582-583. ACM (2002) 
38. Patel, D., Agamanolis, S.: Habitat: awareness of life rhythms over a distance using networked furniture. In: Adjunct Proceedings of UbiComp 2003 Fifth International Conference on Ubiquitous Computing. Citeseer, Seattle (2003)

39. Tsujita, H., Siio, I., Tsukada, K.: Syncdecor: appliances for sharing mutual awareness between lovers separated by distance. In: CHI 2007 Extended Abstracts on Human Factors in Computing Systems, pp. 2699-2704. ACM (2007)

40. Gooch, D., Watts, L.: The magic sock drawer project. In: Proceedings of the 2011 Annual Conference Extended Abstracts on Human Factors in Computing Systems, pp. 243-252. ACM (2011)

41. Grivas, K.: Digital selves: Devices for intimate communications between homes. Personal and Ubiquitous Computing 10(2-3), 66-76 (2006)

42. Kaye, J.: Love, ritual and videochat. In: The Connected Home: The Future of Domestic Life, pp. 185-202 (2011)

43. Tsakiris, M., Haggard, P.: The rubber hand illusion revisited: visuotactile integration and self-attribution. Journal of Experimental Psychology: Human Perception and Performance 31(1), 80 (2005)

44. Kuchinskas, S.: The Chemistry of Connection: How the Oxytocin Response Can Help You Find Trust, Intimacy, and Love. New Harbinger Publications Incorporated (2009)

45. Misawa, K., Ishiguro, Y., Rekimoto, J.: Livemask: A telepresence surrogate system with a face-shaped screen for supporting nonverbal communication. In: Proceedings of the International Working Conference on Advanced Visual Interfaces, pp. 394397. ACM (2012)

46. Yim, J., Shaw, C.: Design considerations of expressive bidirectional telepresence robots. In: Proceedings of the 2011 Annual Conference Extended Abstracts on Human Factors in Computing Systems, pp. 781-790. ACM (2011) 